\title{
Amortecimento de Ondas Gravitacionais por Campos de Vegetação Flexível Parte 2: Experimentos e Modelação
}

\author{
Sávio Freire Lima; Claudio Freitas Neves \\ Programa de Engenharia Oceânica/COPPE/UFRJ - Rio de Janeiro, RJ \\ savio@lycos.com; neves@peno.coppe.ufrj.br \\ Nara Maria Luzzi Rosauro \\ Instituto de Pesquisas Hidráulicas/UFRGS - Porto Alegre, RS \\ nara.rosauro@ufrgs.br
}

Recebido: 14/11/06 - revisado: 20/04/07 - aceito: 11/05/07

\begin{abstract}
RESUMO
A primeira parte deste trabalho apresentou uma nova abordagem para a determinação do amorteci-mento de ondas de gravidade propagando-se sobre campos de vegetação, que evita as simplificações implicitas em outros modelos apresentados na literatura, os quais não se aplicam a espécies de grande flexibilidade. Para verificação do modelo foram realizados ensaios em canal de ondas utilizando cabos flexiveis de nylon, com $6 \mathrm{~mm}$ de diâmetro, presos ao fundo e mantidos na posição vertical por ação do empuxo. Uma técnica de medi-ção indireta por meio de filmagem em vídeo foi desenvolvida particularmente para o procedimento de calibração do modelo. Ensaios de laboratório foram realizados para diferentes características de onda e de espaçamento entre hastes. Através da análise dimensional, as informações obtidas com o modelo calibrado forneceram uma expressão que relaciona a força média exercida pelo fluido com parâmetros adimensionais do escoamento e da haste. A inserção desta força na equação do movimento integrada na vertical e promediada no tempo como um termo de resistência possibilitou a determinação do decaimento da altura da onda ao longo de um campo de hastes e apontou para a importância da interação entre as mesmas no amortecimento total.
\end{abstract}

Palavras-chave: ondas gravitacionais; amortecimento total.

\section{INTRODUÇÃO}

Na primeira parte deste trabalho foi apresentada uma revisão bibliográfica sobre o amortecimento de ondas por vegetação. Os trabalhos podem ser divididos nas seguintes categorias, quanto à inclusão dos efeitos da vegetação sobre o escoamento:

1. como uma camada de grande viscosidade;

2. como uma forma de atrito de fundo;

3. como forças de arraste sobre estruturas rígidas;

4. como uma lei de dissipação de energia.

As três primeiras abordagens trabalham com a equação da quantidade de movimento e a quarta, com a equação de energia. Deve-se ainda mencionar que uma outra vertente de pesquisa dedica-se ao comportamento hidro-elástico das plantas (Vasco, 2005).
Foram destacados os trabalhos de Dalrymple et al. (1984) e Kobayashi et al. (1993). No primeiro trabalho, os autores utilizam uma lei quadrática para a força de arraste, e obtém uma expressão para a taxa de dissipação de energia onde aparece um termo cúbico da altura da onda. No segundo trabalho, os autores utilizam uma lei linear para o arraste, e ao substituíla na equação do balanço de energia, chegam a uma expressão com decaimento exponencial. Também foi apresentada uma tabela com um resumo das principais características dos estudos efetuados por Dalrymple et al. (1984), Kobayashi et al. (1993), Mendez \& Losada (1999), Mendez \& Losada (2004) e Lima (2005).

Todos os modelos apresentados na primeira parte deste trabalho foram verificados experimentalmente, com bons resultados para as características ensaiadas por seus autores. Em todos eles, porém, o movimento da haste era desprezado. Tal simplificação restringe a aplicação daqueles desenvolvimentos na determinação do amortecimento de ondas na presença de estruturas de grande flexibilidade. 
A abordagem proposta neste trabalho para a solução do problema contempla o deslocamento das hastes e a contribuição da velocidade relativa no cálculo dos esforços exercidos sobre o escoamento. As propriedades hidrodinâmicas (velocidade e aceleração) do escoamento são calculadas pela Teoria da Função de Corrente (não linear) de Dean (1968). O modelo de amortecimento considera a grande flexibilidade das estruturas e o arraste é determinado por uma expressão quadrática. No cálculo da força total, a interação entre hastes é parametrizada por um coeficiente que depende da esbeltez da onda, da profundidade relativa, do diâmetro das hastes e do seu comprimento submerso.

A maior parte dos trabalhos procura determinar somente o efeito final do conjunto de hastes sobre a onda, representando este efeito através de um único parâmetro, o coeficiente de arraste global, e admite que o efeito do conjunto seja dado pela soma das resistências individuais. Particularmente no caso de espécies flexíveis, porém, onde os grandes deslocamentos favorecem o contato direto entre as hastes, novos esforços podem surgir modificando o comportamento idealizado.

A modelagem de uma haste isolada, que possibilita a determinação precisa dos coeficientes relacionados às forças atuantes localmente, torna possível a consideração explícita de um termo de interação entre hastes na equação final do amortecimento pelo campo. Esta perspectiva traz consigo uma abordagem inédita para este tipo de estudo.

\section{MODELO PROPOSTO}

O modelo que representa o efeito do campo de vegetação sobre o escoamento utiliza as equações do movimento promediadas no tempo e na vertical, onde introduz-se um termo de resistência $\overline{\mathrm{F}}_{\mathrm{x}}$ causada pelas hastes flexíveis. Este modelo, denominado de "modelo de campo", é regido pela equação:

$$
\begin{aligned}
& \mathrm{H} \frac{\partial \mathrm{H}}{\partial \mathrm{x}}= \\
& \quad-\frac{8}{\rho_{\mathrm{f}} \mathrm{g}(4 \mathrm{n}-1)}\left[\frac{1}{4} \rho g \mathrm{H}^{2} \frac{\partial \mathrm{n}}{\partial \mathrm{x}}+\rho_{\mathrm{f}} \mathrm{g}(\mathrm{h}+\bar{\eta}) \frac{\partial \bar{\eta}}{\partial \mathrm{x}}+\overline{\mathrm{F}}_{\mathrm{x}}\right]
\end{aligned}
$$

onde $\mathrm{H}$ é a altura da onda, h é a profundidade local (fundo horizontal), n é a relação entre celeridade de grupo e da onda, $\bar{\eta}$ é o nível médio do canal, incluindo o rebaixamento ("set-down") ou elevação ("set-up") não linear até $\mathrm{O}(\mathrm{H})^{2} ; \rho_{\mathrm{f}}$ é a massa specífica do fluído, g é a aceleração da gravidade, h é a profundidade do canal e e $\overline{\mathrm{F}}_{\mathrm{x}}$ foi introduzido para representar forças promediadas no período da onda que estejam sendo exercidas sobre a coluna d'água, como por exemplo a reação exercida pelas hastes.

A força total exercida sobre o fluido por $\mathrm{u}$ nidade de área horizontal é calculada em termos da força exercida por uma única haste e da quantidade de hastes por unidade de área pela relação:

$\overline{\mathrm{F}}_{\mathrm{x}}=\alpha \mathrm{N}_{\mathrm{h}} \cdot \overline{\mathrm{F}}_{\mathrm{x}}^{1}$

onde $\alpha$ é um coeficiente de interação entre hastes, $\mathrm{N}_{\mathrm{h}}$ é o número de hastes por metro quadrado e $\overline{\mathrm{F}}_{\mathrm{x}}^{1}$ é numericamente igual à força média exercida ao longo da coluna d'água durante a passagem de uma onda. A priori, não se impõe que a resistência do campo seja a soma dos efeitos individuais das hastes.

A haste flexível, menos densa que a água e posta em movimento pelas ondas, é discretizada em um número de nós escolhido pelo modelador. Em cada segmento, utiliza-se a equação de Morrison modificada, para considerar o movimento da estrutura (Brebia \& Walker, 1979):

$\overrightarrow{\mathrm{F}}_{\mathrm{T}}=\frac{1}{2} \underbrace{\mathrm{C}_{\mathrm{D}} \rho_{\mathrm{f}} \overrightarrow{\mathrm{A}}_{\mathrm{eq}} \overrightarrow{\mathrm{u}}_{\mathrm{rel}}\left|\overrightarrow{\mathrm{u}}_{\mathrm{rel}}\right|}_{(1)}+\underbrace{\mathrm{k}_{\mathrm{m}} \rho_{\mathrm{f}} \mathrm{V}_{\mathrm{el}} \overrightarrow{\mathrm{a}}_{\mathrm{rel}}}_{(2)}+\underbrace{\rho_{\mathrm{f}} \mathrm{V}_{\mathrm{el}} \overrightarrow{\mathrm{a}}_{\mathrm{f}}}_{(3)}$

onde $C_{D}$ é o coeficiente de arraste, $\vec{A}_{\text {eq }}$ corresponde à área da face da estrutura normal ao vetor velocidade relativa entre o fluido e o nó, $\overrightarrow{\mathrm{u}}_{\text {rel }}=\overrightarrow{\mathrm{u}}_{\mathrm{f}}-\overrightarrow{\mathrm{u}}_{\mathrm{v}}$ é a velocidade relativa entre o fluido e a haste, $k_{m}$ é o coeficiente de massa adicional e $\vec{a}_{\text {rel }}=\vec{a}_{f}-\vec{a}_{v}$ é a aceleração relativa. $\mathrm{O}$ primeiro termo da equação (3) corresponde à força de arraste e os dois outros termos correspondem à força de inércia, que por conveniência foi desmembrada em duas parcelas. As velocidades e acelerações relativas são calculadas para cada nó por diferenças finitas, conforme descrito na Parte I do artigo.

A Figura 1 apresenta um resumo da metodologia proposta e das diferentes etapas de sua aplicação. 


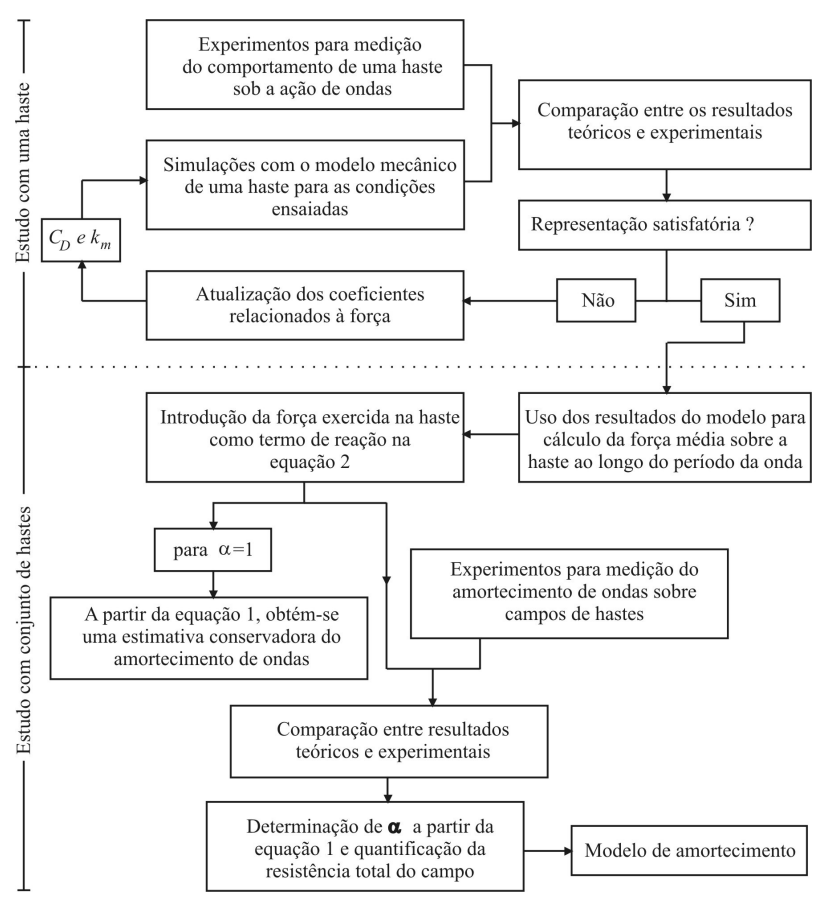

Figura 1 - Metodologia proposta para investigação e modelagem do amortecimento ocasionado pelo conjunto de uma determinada haste.

\section{PROCEDIMENTOS EXPERIMENTAIS}

As atividades de laboratório utilizaram hastes artificiais cujo comportamento hidrodinâmico assemelhava-se ao da espécie vegetal existente no reservatório de Ilha Solteira, conforme observado por filmagens subaquáticas em maio de 2003. A seleção do material foi feita com base em dois critérios fundamentais: (i) assim como as hastes das plantas mais desenvolvidas, a haste artificial deveria possuir baixa resistência à flexão, a ponto de somente permanecer na posição vertical quando submetida à ação do empuxo; (ii) sob a ação de ondas, com altura e período semelhantes aos registrados no reservatório paulista, o elemento deveria responder com deslocamentos de magnitude próxima à observada nas espécies nativas. Após um processo de teste com diferentes materiais disponíveis comercialmente, optou-se pelo uso de cabos de nylon trançado com $6 \mathrm{~mm}$ de diâmetro e massa específica $\rho_{\mathrm{v}}=681,43 \mathrm{~kg} / \mathrm{m}^{3}$. Este elemento satisfez as condições impostas, sendo ainda de fácil manipulação.

Todas as atividades de laboratório foram realizadas nas instalações do Instituto de Pesquisas Hidroviárias/INPH no Rio de Janeiro, em três etapas distintas. Os ensaios para calibração do modelo numérico para uma única haste foram conduzidos entre os meses de maio e junho de 2004. Os ensaios com campo de hastes foram realizados entre os meses de janeiro e fevereiro de 2005 para um espaçamento entre elementos de $50 \mathrm{~mm}$ e, em março do mesmo ano, para um espaçamento de $25 \mathrm{~mm}$.

O canal possui $43 \mathrm{~m}$ de comprimento por $0,8 \mathrm{~m}$ de largura e $1 \mathrm{~m}$ de profundidade. $\mathrm{O}$ gerador de ondas acoplado é de acionamento hidráulico, operando com um pistão controlado por micro computador. Na extremidade do canal oposta ao batedor, há uma praia absorvedora feita com brita de granulometria variada, com 6 m de extensão.

\section{Medições do comportamento de uma haste}

A calibração do modelo de uma haste utilizou uma técnica de medição do movimento com vídeo. Pontos discretos foram marcados ao longo do elemento com tinta fosforescente a um espaçamento regular de $5 \mathrm{~cm}$. O processamento da posição dos nós ao longo do tempo forneceu o histórico de velocidades da haste. Comparando-se as curvas de posição e velocidade com aquelas geradas pelo modelo numérico dado pelas equações 15, 16 e 18 (Parte I do artigo), pôde-se quantificar os coeficientes de força $\left(\mathrm{C}_{\mathrm{D}}\right)$ utilizados nas simulações numéricas.

Durante os ensaios, uma lâmpada ultravioleta compacta L.C. Light de $25 \mathrm{~W}$ foi utilizada para realçar a marcação dos pontos. A movimentação da haste durante a incidência de ondas foi filmada através das janelas de observação do canal por uma câmera de vídeo modelo JVC GR-D72U a uma freqüência de aquisição de 29,97 quadros por segundo. Os dados foram armazenados em formato digital em mídia MDV. O tempo de filmagem para cada ensaio foi de um minuto. A Figura 2 apresenta um exemplo da imagem registrada em dois quadros de um mesmo experimento.

(A)
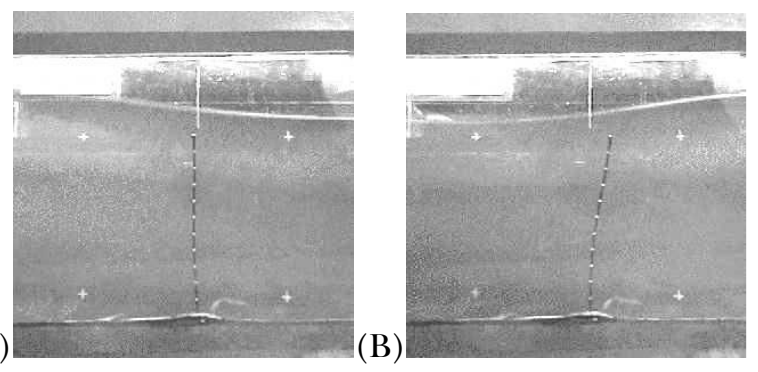

Figura 2 - Quadros de filmagem. A) haste graduada em seu recuo máximo após a passagem da cava da onda. B) Haste em sua posição mais avançada após passagem da crista da onda. 
O processamento das informações obtidas foi dividido em três etapas: (i) conversão dos dados para vídeo padrão Microsoft Windows em formato AVI com resolução de $720 \times 480$ de 24 bits com Codec Cinepak, através de uma placa de captura Matrox modelo RT2000; (ii) edição dos arquivos de vídeo através do programa Adobe Premier ${ }^{\circledR}$, de modo a selecionar dentro do tempo de filmagem do ensaio, o intervalo a ser estudado; (iii) identificação da posição das marcas em cada quadro da filmagem com o auxilio do programa ONDAS desenvolvido por Moacyr de Paula do Laboratório de Multimídia do NCE/UFRJ.

A conversão das informações em unidade de píxel para uma escala métrica foi feita com base em marcações no vidro do canal, cujas distâncias eram precisamente conhecidas. A Figura 3 apresenta o esquema principal de posicionamento da câmera, bem como um exemplo do resultado obtido após a digitalização.

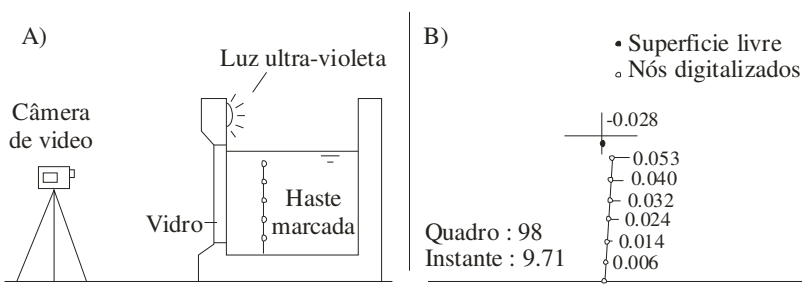

Figura 3- Técnica de filmagem utilizada para obtenção da posição dos nós da haste. A) Seção do canal de ondas utilizada para registrar o movimento da haste. B) Resultado obtido para um quadro digitalizado.

Para garantir a precisão da metodologia, as posições dos nós obtidas devem passar por um procedimento de correção que leva em conta as distorções ópticas (Lima, 2005).

As condições físicas dos ensaios procuraram reproduzir condições semelhantes às registradas no ambiente natural em escala 1:1. Foram usados dois níveis d'água, correspondendo a 0,75 e 0,55 m de profundidade. As alturas de onda consideradas para estudo foram de aproximadamente 0,10, 0,15 e 0,20 $\mathrm{m}$, com períodos de 1,0, 1,5 e 2,0 segundos, perfazendo um total de 12 experimentos. Os comprimentos das hastes foram sempre $0,1 \mathrm{~m}$ menores que a lâmina d'água, de forma que, mesmo diante da maior onda, a haste permanecesse sempre submersa, evitando o aparecimento de outros fenômenos decorrentes da redução de empuxo. As condições detalhadas de ensaio são descritas por Lima (2005).
Antes de cada ensaio, a haste de nylon era recolocada manualmente na posição vertical, garantindo assim que as séries de posição dos nós possuíssem sempre a mesma origem. Tal procedimento se fazia necessário para algumas das ondas de maior comprimento pois, após induzirem oscilação nas hastes, estas estabilizavam em posição inclinada. Para garantir condição inicial de repouso, foi dado um intervalo de 10 minutos entre cada ensaio.

\section{Medições com campo de hastes}

A Figura 4 apresenta o esquema de montagem e medição dos experimentos no canal. O campo foi colocado a barlamar das janelas de vidro, com o intuito de aumentar o tempo de trabalho antes da chegada de ondas refletidas pela praia.

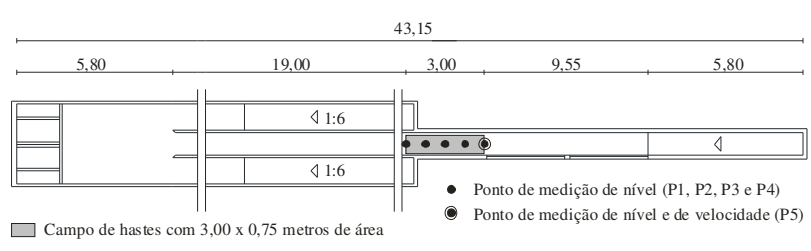

Figura 4 - Vista superior do canal de ondas mostrando configuração do aparato experimental para ensaios com campo de haste.

Para os experimentos com campo de hastes, a fixação no fundo foi garantida através de telas de fer-ro com 3,4 mm de diâmetro (Fio 10) e espaçamento entre nós $25 \mathrm{~mm}$. Foram montadas 3 grades de $1,0 \times 0,75 \mathrm{~m}$ e cada uma delas foi colocadas no fundo do canal, lado a lado, de forma a representar um campo com $3 \mathrm{~m}$ de extensão por $0,75 \mathrm{~m}$ de largura. Em uma primeira montagem, os cabos foram fixados a $5 € \mathrm{~cm}$ de distância um do outro $\left(\mathrm{E}_{\mathrm{v}}=5,0 \mathrm{~cm}\right)$, configurando uma densidade $\mathrm{N}_{\mathrm{h}}=1 / \mathrm{E}_{\mathrm{v}}{ }^{2}$ $=400$ hastes $/ \mathrm{m}^{2}$. Na segunda montagem, o espaçamento utilizado entre os elementos foi de $\mathrm{E}_{\mathrm{v}}=2,5 \mathrm{~cm}$, configurando uma densidade $\mathrm{N}_{\mathrm{h}}=1600$ hastes $/ \mathrm{m}^{2}$.

A amarração dos cabos nos nós da grade foi feita por meio de presilhas plásticas com $10 \mathrm{~cm}$ de comprimento por $0,25 \mathrm{~cm}$ de largura. A Figura 5 apresenta o processo de fixação dos cabos na tela de ara-me. Ao contrário dos experimentos com uma única haste, onde o contraste com a tinta fosforescente exigiu cabos escuros, nos experimentos com campo de hastes foram utilizados cabos de coloração branca. Na Figura 6, as peças montadas estão 
posicionadas no canal de ondas para testes preliminares.

As características de onda ensaiadas foram semelhantes às utilizadas na etapa com uma haste, sendo que, por questões operacionais relacionadas à quantidade de material utilizado e ao procedimento de corte e montagem, a altura das hastes manteve-se fixa e igual a $0,6 \mathrm{~m}$. O plano de ensaios utilizado está indicado na Tabela 2.

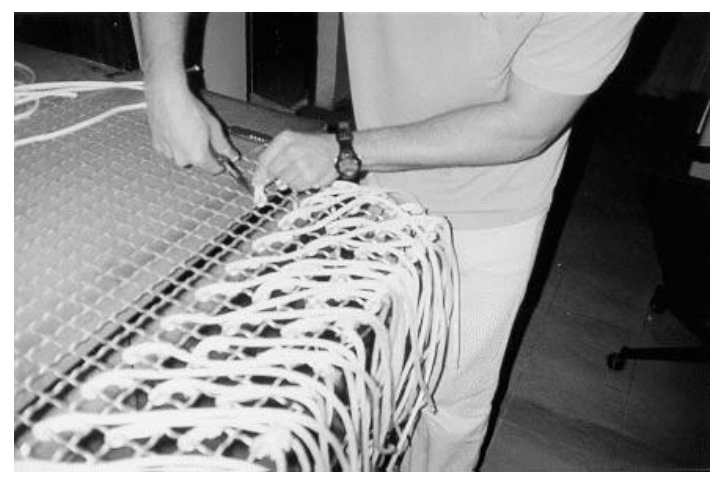

Figura 5 - Preparação para experimentos com campo de hastes através da montagem das telas de 1,00 x $0,75 \mathrm{~m}$ com hastes de $0,6 \mathrm{~m}$.

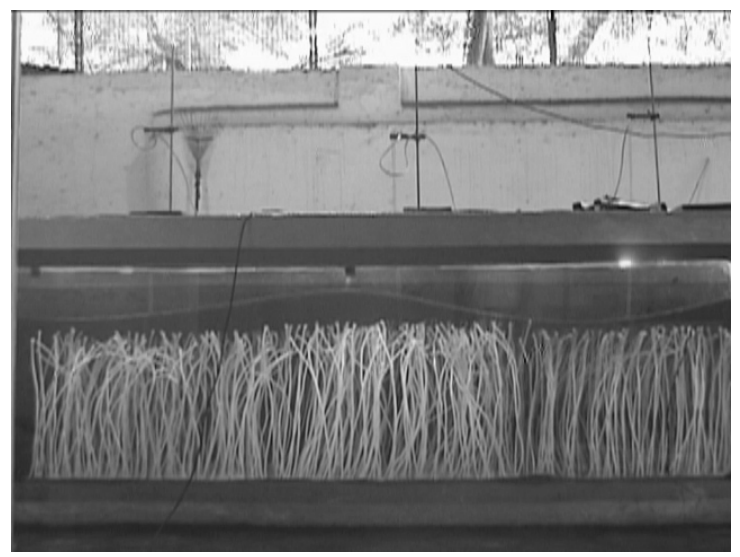

Figura 6 - Testes preliminares no canal de ondas com o campo artificial.

Os experimentos foram realizados em três etapas, a saber: (i) sem a presença do campo artificial, (ii) com 3 metros de campo e uma densidade de 400 hastes $/ \mathrm{m}^{2}$, (iii) com 3 metros de campo e uma densidade de 1600 hastes $/ \mathrm{m}^{2}$. Durante todos os ensaios, as alturas de onda foram registradas ao longo de toda a extensão do campo por meio de cinco sondas resistivas. Dois micro ADV's SonTek de $16 \mathrm{MHz}$ foram utilizados para determinação das componentes das velocidades orbitais em duas elevações fixas de $0,7 \mathrm{~h}$ e $0,5 \mathrm{~h}$, acima do fundo. Procurou-se, desta forma, não só identificar as modificações que a presença da vegetação induzia na altura da onda, mas também no campo de velocidades.

Cada um dos experimentos foi executado três vezes; desta forma, trabalhou-se com valores médios da taxa de decaimento, garantindo uma maior representatividade para os dados e identificando anomalias pontuais no processo de medição. Considerando as nove condições de ondas escolhidas, os ensaios sem vegetação e aqueles para as duas densidades de haste referidas, foram realizados 81 experimentos, todos com medições simultâneas de nível em cinco pontos, e de velocidade em dois pontos na mesma vertical do último sensor.

\section{RESULTADOS}

\section{Comportamento de uma haste}

Cada uma das 12 configurações experimentais apresentadas na Tabela 1 foi simulada pelo modelo numérico para uma haste, tendo inicialmente os coeficientes $C_{D}$ e $k_{m}$ como parâmetros de ajuste. Posteriormente, devido à reduzida magnitude dos termos de inércia, optou-se por modelar a força sobre os nós somente pelo termo de arraste, ou seja, o termo(1) da equação(3). A pequena influência dos demais termos dentro das condições estudadas está relacionada à dependência dos mesmos no volume do elemento, o que os torna uma função quadrática de um diâmetro de 0,006 m.

Tabela 1 - Plano de ensaios utilizado para os estudos com campo de hastes.

\begin{tabular}{llllllll}
\hline $\begin{array}{c}\text { Ensaio }_{(\mathrm{m})} \\
\text { s }\end{array}$ & $\begin{array}{l}\mathrm{H}_{\mathrm{o}} \\
(\mathrm{m})\end{array}(\mathrm{m})$ & $\begin{array}{l}\mathrm{T} \\
(\mathrm{s})\end{array}$ & $\begin{array}{l}\mathrm{L} \\
(\mathrm{m})\end{array}$ & $\mathrm{h} / \mathrm{L}$ & $\mathrm{H} / \mathrm{L}$ & $\begin{array}{l}\phi / \mathrm{L} \\
\left(\mathrm{x} 10^{3}\right)\end{array}$ \\
\hline 01-Ch 0,7 & 0,6 & 0,05 & 1,00 & 1,57 & 0,447 & 0,032 & 3,831 \\
02-Ch 0,7 & 0,6 & 0,05 & 1,25 & 2,34 & 0,299 & 0,021 & 2,564 \\
03-Ch 0,7 & 0,6 & 0,05 & 1,50 & 3,13 & 0,224 & 0,016 & 1,919 \\
04-Ch 0,7 & 0,6 & 0,10 & 1,00 & 1,61 & 0,435 & 0,062 & 3,729 \\
05-Ch 0,7 & 0,6 & 0,10 & 1,25 & 2,37 & 0,295 & 0,042 & 2,533 \\
06-Ch 0,7 & 0,6 & 0,10 & 1,50 & 3,15 & 0,222 & 0,032 & 1,905 \\
07-Ch 0,7 & 0,6 & 0,15 & 1,00 & 1,68 & 0,418 & 0,090 & 3,582 \\
08-Ch 0,7 & 0,6 & 0,15 & 1,25 & 2,42 & 0,290 & 0,062 & 2,483 \\
09-Ch 0,7 & 0,6 & 0,15 & 1,50 & 3,19 & 0,219 & 0,047 & 1,881 \\
\hline
\end{tabular}

As séries temporais de posição e velocidade de cada nó, obtidas pelo tratamento dos dados de 
vídeo, foram tomadas como referência para indicar o grau de representatividade dos dados gerados no modelo numérico. Para cada uma das 12 configurações experimentais, diferentes valores de $C_{D}$ foram usados em simulações numéricas até que se considerasse o resultado do modelo coerente com os valores experimentais. O coeficiente de determinação, $R^{2}$, foi escolhido como parâmetro de referência da qualidade do ajuste por fornecer uma estimativa do percentual da variação da medição experimental que o modelo pode explicar.

Para todas as situações consideradas, os intervalos de amostragem selecionados para as comparações com os dados de laboratório consistiram em três períodos de onda. $\mathrm{O}$ inicio do intervalo de comparação foi estabelecido como sendo após a fase transiente de movimentação da haste. Durante a fase inicial, a haste (que parte do repouso) apresenta um comportamento irregular, com a magnitude crescente de suas oscilações. Em geral, depois de decorridos 3 a 6 períodos de onda do início da simulação, o movimento torna-se harmônico e os três ciclos seguintes podem ser selecionados para a comparação com os dados experimentais.

Os resultados numéricos foram obtidos através da implementação do modelo em MatLab ${ }^{\circledR}$ (MathWorks, Inc.). Para uma execução padrão, com passo de tempo de 0,001 segundos, o tempo computacional necessário para calcular 15.000 passos de tempo, ou 15 segundos no modelo físico, foi da ordem de 15 minutos em um computador tipo PC Athlon XP 2.200 MHz com $256 \mathrm{MB}$ de memória RAM. Uma interface gráfica foi desenvolvida em Visual Basic de modo a possibilitar leitura dos arquivos de dados, animação dos resultados e exportação de curvas comparativas tanto do modelo numérico quanto dos resultados experimentais. A Figura 7 apresenta um exemplo dos resultados gerados pelo modelo calibrado para o experimento $09-1 \mathrm{~h}$, mostrando as curvas de superfície livre, posição e velocidade dos nós.

Embora as ondas no modelo teórico fossem regulares (porém não lineares), as ondas geradas no canal apresentaram flutuações de forma em relação aos valores previstos. Observa-se nos gráficos que pequenas variações, principalmente relacionadas à amplitude, são registradas entre cristas ou cavados adjacentes. Em alguns dos experimentos, observa-se que, mesmo com a superfície livre numérica estando em fase com a superfície livre experimental, ainda ocorre alguma defasagem entre o movimento dos nós previsto e medido (máxima de $20 \%$ do período). Conseqüentemente, as velocidades também se apresentarão fora de fase. Em tais casos, a adoção de um valor para o coeficiente de arraste diferente do apontado, somente possibilitou alteração na magnitude das componentes, não na defasagem. Testes numéricos complementares, com diferentes passos de tempo, indicaram que a adoção de outros valores não melhoraram os resultados.
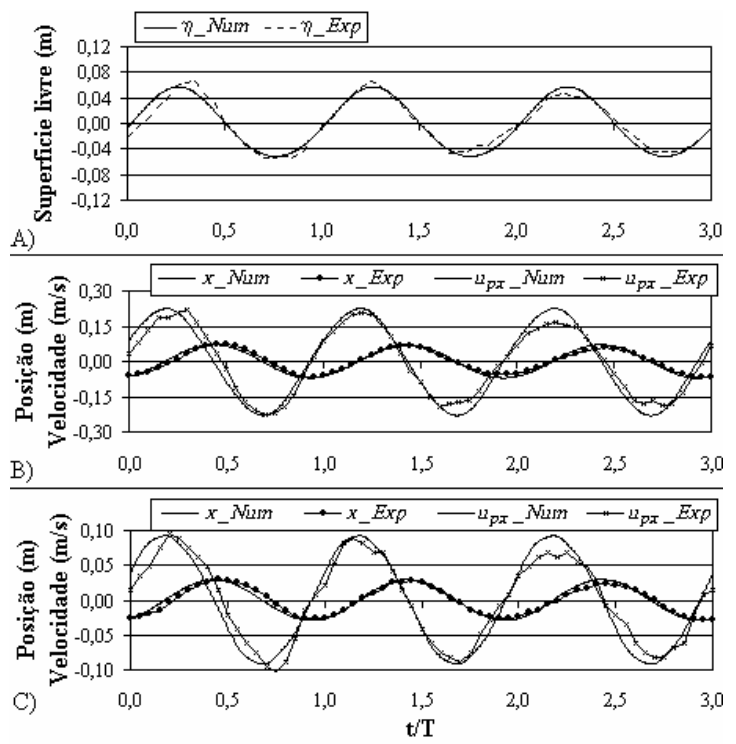

Figura 7 - Comparação entre os dados experimentais do ensaio 09-1h e os resultados numéricos obtidos para $C_{D}=0,05$. A) Posição da superfície livre sobre o eixo inicial da haste. B) Posição e velocidade do nó superior $(n=6)$ e C) do nó central $(n=3)$.

Do ponto de vista exclusivamente mecânico, as defasagens registradas não devem influenciar significativamente a estimativa da força média exercida sobre um elemento. Tomando-se a força resultante em um dado instante como a variação da quantidade de movimento do elemento, é razoável considerar que a força exercida pelo escoamento que é predominantemente horizontal — pode ser obtida a partir do conhecimento de sua massa, e da curva de velocidades horizontais. Assim, como o modelo forneceu curvas de velocidade com períodos e amplitudes muito semelhantes às medidas, o valor médio da força estará próximo do valor real, não sofrendo influência da defasagem.

É possível que a introdução dos termos inerciais no cálculo das forças reduza a diferença de fase, o que implicaria em ligeiras modificações nos valores de $C_{D}$ encontrados. No âmbito desta linha de pesquisa, no entanto, julga-se satisfatória a representação da força somente pelo termo de arraste, dado 
que se conhece uma estimativa da baixa representatividade do termo ignorado. Por este motivo, tomouse uma postura menos rigorosa na interpretação do critério de julgamento, onde foi considerado satisfatório aquele ajuste com $\mathrm{R}^{2}>0,5$.

O modelo numérico, contudo, foi implementado com a expressão completa para força, com todos os seus termos, fornecendo uma ferramenta para trabalhos mais aprofundados nesta questão. Uma investigação futura poderia partir dos coeficientes de arraste aqui apresentados, procurando melhorar $\mathrm{o} \quad \mathrm{R}_{\mathrm{m}}^{2}$ de cada experimento através da variação de $\mathrm{k}_{\mathrm{m}}$.

Os valores de $C_{D}$ obtidos para cada um dos experimentos foram expressos em função do número de Reynolds, $\operatorname{Re}_{\mathrm{rel}}=\left(\mathrm{u}_{\mathrm{rel}}\right)_{\max } \phi / \nu$ e do parâmetro de Keulegan-Carpenter, $\mathrm{KC}_{\mathrm{rel}}=\left(\mathrm{u}_{\mathrm{rel}}\right)_{\max } \mathrm{T} / \phi$, onde $\checkmark$ é a viscosidade cinemática do fluido, $\phi$ é o diâmetro da haste, $\mathrm{T}$ é o período da onda, e $\mathrm{u}_{\mathrm{rel}}$ é a velocidade da haste em relação à velocidade do escoamento. Os resultados estão ilustrados nas Figuras 8 e 9.

O número de Keulegan-Carpenter, que represen-ta uma relação entre a excursão da partícula de fluido e o diâmetro do cilindro, foi proposto em 1958 por esses pesquisadores ao investigarem as forças exercidas por ondas estacionárias sobre um cilindro vertical e uma placa plana. Ao colocarem traçadores na água, eles puderam visualizar a formação de vórtices e relacioná-la com este novo parâmetro que serve como indicador do descolamento dos filetes: para valores baixos, não se verifica a separação, para valores na vizinhança de 15 , um único vórtice é formado, enquanto que para valores mais altos, identifica-se a formação de uma esteira de vórtices. A descoberta importante de Keulegan e Carpenter, confirmada por vários pesquisadores posteriormente (e.g. Chakrabarti, 1987) foi que o coeficiente de arraste $C_{D}$ não se correlacionava bem com o número de Reynolds. Conforme estudos e observação de Wiegel (1964, p.258-259), no caso de escoamentos oscilatórios, não existe uma relação clara entre os coeficientes hidrodinâmicos $\left(\mathrm{C}_{\mathrm{M}}\right.$ e $\left.\mathrm{C}_{\mathrm{D}}\right)$ e o número de Reynolds, ao contrário do que ocorre em escoamentos permanentes. Segundo Wiegel, a relação com o Re somente é válida quando o tamanho dos vórtices é pequeno em relação ao diâmetro do obstáculo, fato este que não se observou nos experimentos aqui descritos.

Como se pode verificar nas Figuras 8 e 9, o coeficiente $C_{D}$ apresentou melhor relação de dependência com o parâmetro de KeuleganCarpenter, o que está de acordo com Mendez \&
Losada (2004). As magnitudes aqui encontradas são superiores às obtidas por aqueles autores, o que se atribui à inclusão do movimento das hastes, pois, tradicionalmente, calcula-se a força usando a velocidade do fluido em vez da velocidade relativa e portanto um coeficiente de arraste menor. Em geral os valores de $C_{D}$ encontrados para escoamentos oscilatórios são bem mais elevados do que os obtidos no caso de escoamentos permanentes. Por exemplo, Wiegel (1964) apresenta valores na faixa 0,1 a 6 para Re na faixa de $10^{3}$ a $10^{6}$. Stansby et al. (1983), Sarpkaya (1984), Cotter e Chakrabarti (1984) e Zdravicovich (1984) relatam valores na faixa 1,25 a 2,25 para KC na faixa de 10 a 20. Além disso, é razoavelmente bem estabelecido que o valor de $C_{D}$ é significativamente influenciado pela rugosidade relativa (Zdravicovich, 1984), e portanto, deve-se levar em conta o fato de que as hastes usadas no presente estudo (cabos de nylon) eram muito rugosas. A bibliografia sobre o assunto é extensa e a escolha de valores de projeto para $C_{D}$ em escoamentos oscilatórios requer muito cuidado.

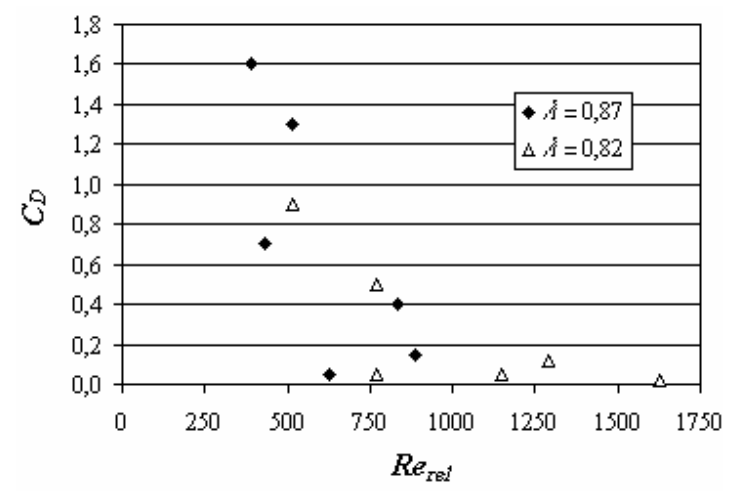

Figura 8 - Valores calibrados do coeficiente de arraste $C_{D}$ como função do número de Reynolds para velocidade relativa nos dois grupos de $\lambda$ utilizados $(\lambda=s / h)$.

No cálculo de $C_{D}$, a velocidade relativa máxima $\left(\mathrm{u}_{\mathrm{rel}}\right)_{\max }$ não é uma grandeza prática para se trabalhar, pois requer o conhecimento do histórico de velocidades da haste e do fluido ao longo de todo um período de onda. Adiante, será apresentada uma expressão mais conveniente, onde o valor de $C_{D}$ é expresso em função de grandezas mensuráveis diretamente no experimento.

A Tabela 2 apresenta os resultados obtidos para as simulações. A quarta coluna apresenta um parâmetro adimensional da força média calculada 
que adiante será usado para a obtenção de uma expressão geral para sua determinação.

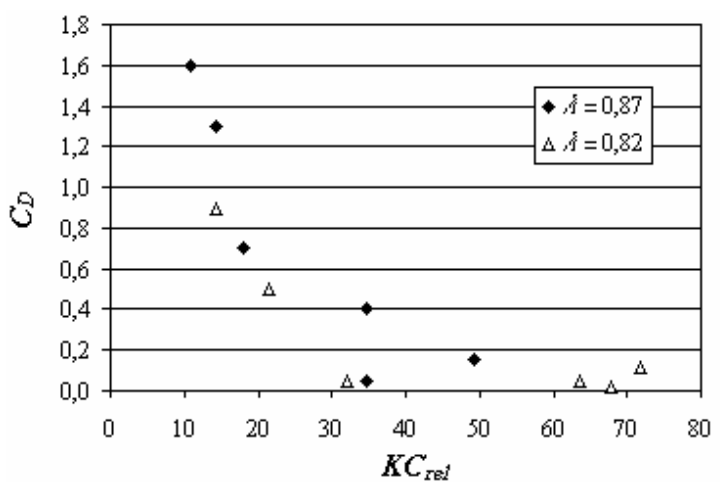

Figura 9 - Valores calibrados do coeficiente de arraste como função do parâmetro de Keulegan-Carpenter para velocidade relativa.

Os valores $\overline{\mathrm{F}}_{\mathrm{x}}^{1}$ podem ser substituídos diretamente na equação (2), onde para $\alpha=1$, obtém-se uma primeira estimativa do termo de resistência por metro quadrado decorrente da presença das hastes. Para um fundo plano e horizontal, a equação (1) pode ser reescrita como:

$H \frac{\partial}{\partial x} H=-\frac{8 \alpha N_{h} \bar{F}_{x}^{1}}{\rho_{f} g(4 n-1)}$

onde a variação da posição média da superfície livre no sentido de propagação da onda foi considerada desprezível. Estimativas preliminares realizadas para as ondas ensaiadas mostraram que estas variações são da ordem de décimos de milímetros.

da

Ao se substituir os valores da terceira coluna

Tabela 2 na equação (4), a expressão pode ser integrada, e o decaimento em altura de onda para as características do experimento será dado por:

$\frac{\mathrm{H}}{\mathrm{H}_{\mathrm{o}}}=\sqrt{1-\kappa \mathrm{x}}$

onde $\kappa=\frac{16 \alpha N_{h} \bar{F}_{x}^{1}}{\rho_{f} g(4 n-1)}$

A aplicação desta expressão fica restrita aos casos ensaiados onde o modelo representou satisfa- toriamente o comportamento da haste. Com o intuito de se obter uma expressão mais geral para o amortecimento, procurou-se estabelecer uma equação para representar $\overline{\mathrm{F}}_{\mathrm{x}}^{1}$ a partir de grandezas diretas do ensaio. Uma vez obtida uma expressão representativa, evita-se a necessidade de novas calibrações do modelo para condições que estejam dentro dos limites de validade utilizados na sua determinação.

Tabela 2 - Força média exercida pela haste sobre a coluna d'água durante a passagem de uma onda.

\begin{tabular}{llll}
\hline Ensaio & $\mathrm{R}^{2}{ }_{\mathrm{m}}$ & $\overline{\mathrm{F}}_{\mathrm{x}}^{1}(\mathrm{~N})$ & $\overline{\mathrm{F}}_{\mathrm{x}}^{1} \mathrm{~T}^{2} / \rho_{\mathrm{v}} \mathrm{s}^{4}$ \\
\hline $07-1 \mathrm{~h}$ & 0,52 & $6,45 \mathrm{E}-04$ & $5,30 \mathrm{E}-06$ \\
$08-1 \mathrm{~h}$ & 0,54 & $2,61 \mathrm{E}-04$ & $4,82 \mathrm{E}-06$ \\
$09-1 \mathrm{~h}$ & 0,96 & $4,72 \mathrm{E}-05$ & $1,55 \mathrm{E}-06$ \\
$10-1 \mathrm{~h}$ & 0,44 & $1,13 \mathrm{E}-03$ & $9,30 \mathrm{E}-06$ \\
$11-1 \mathrm{~h}$ & 0,82 & $9,21 \mathrm{E}-04$ & $1,70 \mathrm{E}-05$ \\
$12-1 \mathrm{~h}$ & 0,83 & $4,30 \mathrm{E}-04$ & $1,41 \mathrm{E}-05$ \\
$19-1 \mathrm{~h}$ & 0,40 & $4,01 \mathrm{E}-04$ & $1,44 \mathrm{E}-05$ \\
$20-1 \mathrm{~h}$ & 0,17 & $4,83 \mathrm{E}-05$ & $3,89 \mathrm{E}-06$ \\
$21-1 \mathrm{~h}$ & 0,85 & $4,54 \mathrm{E}-05$ & $6,50 \mathrm{E}-06$ \\
$22-1 \mathrm{~h}$ & 0,14 & $4,86 \mathrm{E}-04$ & $1,74 \mathrm{E}-05$ \\
$23-1 \mathrm{~h}$ & 0,24 & $1,04 \mathrm{E}-04$ & $8,39 \mathrm{E}-06$ \\
24-1h & 0,95 & $6,43 \mathrm{E}-04$ & $9,21 \mathrm{E}-05$ \\
\hline
\end{tabular}

A experiência adquirida no processo experimental juntamente com a análise dimensional possibilita-ram estabelecer uma expressão relacionando a força procurada a parâmetros característicos do escoamento e da haste. Propôs-se uma relação funcional entre os parâmetros adimensionais do tipo:

$\left(\frac{\bar{F}_{x}^{1} T^{2}}{\rho_{v} s^{4}}\right)=a\left(\frac{H}{L}\right)^{b}\left(\frac{h}{L}\right)^{c}\left(\frac{\phi}{L}\right)^{d}$

onde a, b, c e d são constantes a determinar; $\rho_{\mathrm{v}}$ é a massa específica da espécie vegetal, e s é a relação entre a altura da haste e a profundidade do canal.

Para obtenção dos coeficientes com base nos valores de $\mathrm{T}, \rho_{\mathrm{v}}, \mathrm{s}, \mathrm{H}, \mathrm{h}, \phi$ e L de cada ensaio, tomaram-se como referência os valores de $\overline{\mathrm{F}}_{\mathrm{x}}^{1}$ gerados pelo modelo numérico e recorreu-se à técnica de regressão linear multivariada. Os coeficientes obtidos foram: $a=0,133, b=3, c=-4$ e $d=1$. A substituição dos coeficientes ajustados na equação (7) fornece uma expressão final para a força, dada por: 
$\overline{\mathrm{F}}_{\mathrm{x}}^{1}=0,133 \frac{\rho_{\mathrm{v}} \phi \lambda^{4} \mathrm{H}^{3}}{\mathrm{~T}^{2}}$

O resultado final passa a ser uma expressão de boa representatividade $\left(\mathrm{R}^{2}=0,95\right)$, onde os expoentes relacionados ao comprimento da onda se anulam. É interessante observar na equação (8) que a força é proporcional ao cubo da altura da onda.

\section{Comportamento do campo de hastes}

A substituição da forma geral da expressão (7) em (4) fornecerá uma expressão para o decaimento da altura da onda dada por:

$$
\mathrm{H}^{1-\mathrm{b}} \frac{\partial}{\partial \mathrm{x}} \mathrm{H}=-\mathrm{a} \frac{8 \alpha \mathrm{N}_{\mathrm{h}} \mathrm{s}^{4}}{\mathrm{gT} \mathrm{T}^{2}(4 \mathrm{n}-1) \mathrm{L}^{\mathrm{b}}}\left(\frac{\rho_{\mathrm{v}}}{\rho_{\mathrm{f}}}\right)\left(\frac{\mathrm{h}}{\mathrm{L}}\right)^{\mathrm{c}}\left(\frac{\phi}{\mathrm{L}}\right)^{\mathrm{d}}
$$

A integração desta expressão dentro da consideração de que $\mathrm{H}=\mathrm{H}_{0}$ para $\mathrm{x}=0$, fornece:

$$
\frac{H}{H_{o}}=\left[1-a \frac{8 \alpha N_{h} s^{4}(2-b)}{g T^{2}(4 n-1) L^{b} H_{o}{ }^{2-b}}\left(\frac{\rho_{\mathrm{v}}}{\rho_{\mathrm{f}}}\right)\left(\frac{\mathrm{h}}{\mathrm{L}}\right)^{\mathrm{c}}\left(\frac{\phi}{\mathrm{L}}\right)^{\mathrm{d}} \mathrm{x}\right]^{\frac{1}{2-b}}
$$

A substituição dos coeficientes a, b, c e d obtidos fornece uma expressão de decaimento do tipo:

$\frac{\mathrm{H}}{\mathrm{H}_{\mathrm{o}}}=\frac{1}{1+\widetilde{\beta}_{\mathrm{x}}}$

onde

$\widetilde{\beta}=1,064 \frac{\alpha \mathrm{N}_{\mathrm{h}} \phi \lambda^{4} \mathrm{H}_{0}}{\mathrm{gT}^{2}(4 \mathrm{n}-1)} \frac{\rho_{\mathrm{v}}}{\rho_{\mathrm{f}}}$

Esta forma de decaimento é a mesma deduzida analiticamente por Dalrymple et al. (1984) considerando as estruturas vegetais como cilindros rígidos e negligenciando o seu movimento. O fato dos dados experimentais terem apontado uma solução similar sugere que a expressão (11) pode ter uma abrangência mais geral em fenômenos de amortecimento de ondas por grupos de elementos esbeltos, bem como suportam a hipótese de uma lei quadrática para a interação fluido-estrutura.

A expressão (12) evidencia os aspectos que influenciam diretamente o fenômeno final investigado por este trabalho, ou seja, a magnitude do amortecimento das ondas. Algumas relações merecem um comentário a parte:
1. O amortecimento cresce com o aumento da densidade relativa da haste $\rho_{\mathrm{v}} / \rho_{\mathrm{f}}$, indicando que a inércia do elemento prevalece sobre o empuxo enquanto força de resistência ao movimento, pois se o empuxo fosse mais significativo, o amortecimento deveria ser maior para uma menor densidade relativa;

2. como seria esperado, quanto maior o diâmetro da haste $(\phi)$, maior a taxa de amortecimento.

3. a relação não linear entre o amortecimento e a parcela da coluna d'água ocupada pela haste $(\lambda)$, definida como a razão entre o seu comprimento (s) e a profundidade de repouso (h), mostra que o amortecimento é fortemente reduzido a partir do momento que a extremidade da haste se afasta da superfície livre. Este estudo não considerou o caso em que as hastes atravessassem a superfície livre $(\lambda>1)$.

4. Ondas de maior período (mais longas) são menos afetadas pela vegetação, numa razão inversa com o quadrado do período; conseqüentemente, o campo de vegetação funciona como um filtro para ondas curtas, que possuem efeito mais danoso sobre a margem.

Para os experimentos com campo descritos na Tabela 2, todos os parâmetros da equação (12) são conhecidos, exceto o coeficiente de interação entre hastes $(\alpha)$. Através da minimização do erro médio quadrático entre as curvas de amortecimento experi-mentais e teóricas, tal coeficiente foi estimado para cada ensaio. A Figura 10 compara as curvas de decaimento medidas e aquelas previstas com o modelo para $\alpha=1$ e para $\alpha$ igual ao valor ajustado. A sigla MECh refere-se ao Modelo Empírico para Conjunto de hastes proposto (equação (11).

Observou-se o significativo incremento na atenuação das ondas com o aumento do número de hastes por metro quadrado de $400 \quad\left(\mathrm{E}_{\mathrm{v}}=0,050 \mathrm{~m}\right)$ para $1600\left(\mathrm{E}_{\mathrm{v}}=0,025 \mathrm{~m}\right)$. No primeiro caso, a redução da altura da onda nos três metros de campo variou de 2,5 a $5 \%$, enquanto que, após o aumento da densidade, a taxa de atenuação passou a variar em torno de $15 \%$. Este valor é bastante expressivo, principalmente se for considerado que a extensão do campo simulado é somente da ordem de um a dois comprimentos de onda. 


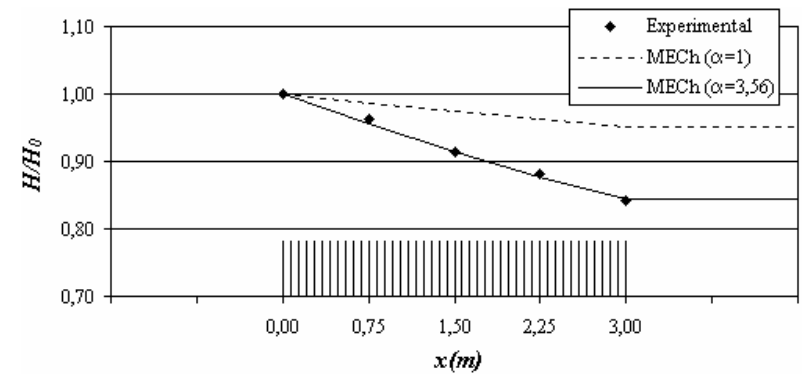

Figura 10 - Decaimento de altura de onda para o experi-

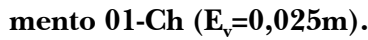

Os valores ajustados para o coeficiente de interação entre hastes estão apresentados na Tabela 3 , onde são indicadas as condições de ondas em cada ensaio. Como seria presumível, $\alpha$ mostrou-se mais representativo nos experimentos com a maior densidade, onde a relação entre a amplitude de oscilação da haste e a distância entre as mesmas torna-se menor, favorecendo o contato direto entre elementos adjacentes.

Observa-se uma constante tendência de crescimento de $\alpha$ com o aumento do período da onda $(\mathrm{T})$, embora seja inversamente proporcional à altura de onda incidente $\left(\mathrm{H}_{\mathrm{o}}\right)$.

Tabela 3 - Coeficiente de interação entre hastes determinado para cada uma das configurações de onda ensaiadas.

\begin{tabular}{|c|c|c|c|c|}
\hline Onda & $\begin{array}{l}\mathrm{H}_{\mathrm{o}} \\
(\mathrm{m})\end{array}$ & $\begin{array}{l}\mathrm{T} \\
(\mathrm{s})\end{array}$ & $\begin{array}{l}\alpha \\
\left(E_{v}=0,050\right)\end{array}$ & $\begin{array}{l}\alpha \\
\left(\mathrm{E}_{\mathrm{v}}=0,025\right)\end{array}$ \\
\hline 01-Ch & 0,05 & 1,00 & 2,309 & 3,563 \\
\hline 02-Ch & 0,05 & 1,25 & 4,553 & 6,320 \\
\hline 03-Ch & 0,05 & 1,50 & 8,789 & 12,237 \\
\hline 04-Ch & 0,10 & 1,00 & 2,102 & 1,865 \\
\hline 05-Ch & 0,10 & 1,25 & 3,396 & 3,293 \\
\hline 06-Ch & 0,10 & 1,50 & 4,604 & 6,124 \\
\hline 07-Ch & 0,15 & 1,00 & 0,694 & 1,291 \\
\hline 08-Ch & 0,15 & 1,25 & 0,756 & 2,507 \\
\hline \multirow[t]{3}{*}{ 09-Ch } & 0,15 & 1,50 & 3,459 & 4,410 \\
\hline & & Média & 3,407 & 4,623 \\
\hline & Desv & Padrão & 2,475 & 3,340 \\
\hline
\end{tabular}

Os valores encontrados sugerem que a resistência ao escoamento imposta pelo conjunto de hastes é em média quatro vezes maior que a estimada pela soma da força individual de cada haste. Este resultado indica que, ao contrário do que se considera na maioria dos desenvolvimentos teóricos propostos na literatura, a interação entre hastes é um fenômeno de grande representatividade e a idéia de se estimar a resistência total do campo pela soma das resistências individuais das hastes não é justificada, principalmente para o caso de hastes flexíveis, onde fenômenos como o entrelaçamento de hastes ou o contato direto podem ocorrer.

Mais uma vez recorre-se à técnica de regressão linear multivariada para determinar uma expressão do comportamento de $\alpha$ em função de parâmetros adimensionais. Incluiu-se a relação entre o diâmetro das hastes e o espaçamento entre as mesmas, fornecendo um conceito análogo à porosidade do campo de hastes. Foram testadas diferentes combinações entre os grupamentos adimensionais, e obteve-se uma função direta e de grande representatividade para o coeficiente de interação entre hastes:

$\alpha=7,44 \frac{\mathrm{L}^{2} \phi^{3 / 2}}{\mathrm{H}_{\mathrm{o}} \mathrm{s}^{2} \mathrm{E}_{\mathrm{v}}{ }^{1 / 2}}$

onde o comprimento de onda utilizado foi estimado pela Teoria da Função de Corrente.

\section{Influência no campo de velocidades}

As medições realizadas com as sondas de velocidade para ensaios com e sem o campo de vegetação tiveram o propósito de responder a duas questões principais: (i) quão representativa é a teoria de onda utilizada no modelo numérico para os ensaios realizados ? (ii) a presença da vegetação introduz alguma outra modificação no campo de velocidades além da redução de sua magnitude ?

Os resultados indicaram que a teoria da função de corrente de $3^{\text {a }}$ ordem representou satisfatoriamente o campo de velocidades das ondas estudadas. A Figura 11 traz as curvas comparativas entre as componentes horizontal e vertical do escoamento obtidas nos dois pontos de medição durante o experimento 03-Ch sem vegetação, e aquelas geradas numericamente. A boa coerência apresentada foi registrada nos demais experimentos.

Comparações similares foram realizadas para as curvas de velocidade obtidas com vegetação. Medições simultâneas de nível no ponto de fixação dos ADV's revelaram que as modificações impostas no campo de velocidades pela presença das hastes estão relacionadas diretamente à redução da altura da onda. Em outras palavras, uma vez conhecida a altura da onda em um ponto do campo, a velocidade orbital neste local pode ser obtida teoricamente. 

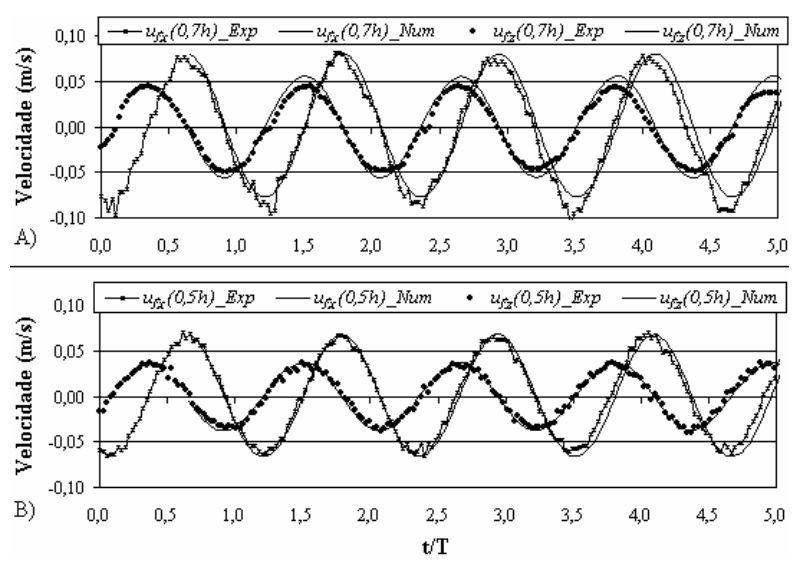

Figura 11 - Comparação entre valores de velocidade do escoamento previsto pela teoria da função de corrente e medidas durante o ensaios 03-Ch. A) Ponto localizado à 0,7h. B) Ponto localizado a $0,5 \mathrm{~h}$.

\section{Modelo de amortecimento}

A discussão conduzida com base nas considerações teóricas e evidências experimentais permite, neste ponto, a proposição de um modelo de amortecimento para ondas de gravidade propagandose em campos formados pelo elemento flexível estudado. A altura da onda poderá ser obtida em qualquer ponto pela expressão:

$\frac{H}{H_{o}}=\frac{1}{1+\widetilde{\beta}_{x}}$

onde a substituição da expressão obtida para $\alpha$ na equação (13) fornece:

$\widetilde{\beta}=7,916 \frac{\mathrm{L}^{2} \lambda^{4}}{\mathrm{gT}^{2}(4 \mathrm{n}-1) \mathrm{s}^{2}} \frac{\rho_{\mathrm{v}}}{\rho_{\mathrm{f}}} \phi^{5 / 2} \mathrm{~N}_{\mathrm{h}}^{5 / 4}$

Desta forma, para uma mesma haste, o amortecimento será uma função somente da profundidade e do comprimento da onda, não dependendo da altura incidente. As Figuras 12 e 13 apresentam as curvas de decaimento para as condições ensaiadas, onde os valores de comprimento de onda utilizados foram estimados pela Teoria da Função de Corrente.

A expressão (15) deve ser aplicada somente em condições próximas às ensaiadas. Uma expressão relacionando as características dos ensaios realizados com os valores encontrados para o coeficiente de arraste é apresentada para fornecer uma primeira estimativa do $\mathrm{C}_{\mathrm{D}}$ a ser utilizado no modelo mecânico em novas simulações com os elementos flexíveis adotados no presente estudo:

$$
\mathrm{C}_{\mathrm{D}}=1,56 \frac{\mathrm{H}^{1 / 2} \mathrm{~h}^{2}}{\phi^{1 / 2} \mathrm{~L}^{2}}
$$

Esta expressão foi obtida através de regressão usando os nove experimentos com $R_{m}^{2}>0,40$. $O$ seu coeficiente de determinação foi $R^{2}\left[C_{D}\right]=0,93$.

A título de comparação, os valores estimados para $\mathrm{C}_{\mathrm{D}}$ foram inseridos na expressão estabelecida por Dalrymple et al. (1984). Os coeficientes de amortecimento, $\beta$, obtidos com esta expressão, mostraram-se até 5 vezes maiores que os valores determinados para $\widetilde{\beta}$. Em uma primeira análise, seria intuitivo supor que, ao considerar-se a flexibilidade (e conseqüentemente o movimento) das hastes, as forças atuantes seriam reduzidas e, desta forma, a resistência ao escoamento se mostrasse menor, o que justificaria o resultado encontrado. Contudo, esta comparação tem um cunho meramente ilustrativo, dado que o modelo proposto neste trabalho, entre outros aspectos, foge da idéia de se atribuir um $\mathrm{C}_{\mathrm{D}}$ único para todo o campo, trabalhando diretamente com uma expressão para a força, que é função da altura da onda.

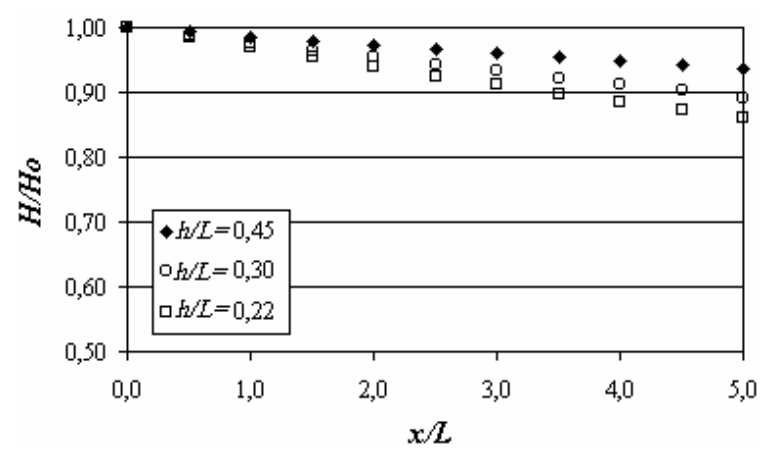

Figura 12 - Decaimento da altura da onda propagando-se através de um campo de hastes flexíveis com espaçamento $\mathbf{E}_{\mathrm{v}}=\mathbf{0 , 0 5 0} \mathrm{m}$.

Além disso, o modelo apresentado contempla uma expressão empírica para representar o efeito de conjunto das hastes, o que não acontece no referido trabalho. Mesmo que este efeito fosse negligenciado através da adoção de um valor unitário para $\alpha$, o uso da velocidade relativa entre a haste e o fluido no cálculo da resistência individual já consiste em uma diferença teórica grande o suficiente para 
limitar a comparação direta entre os dois coeficientes de amortecimento propostos.

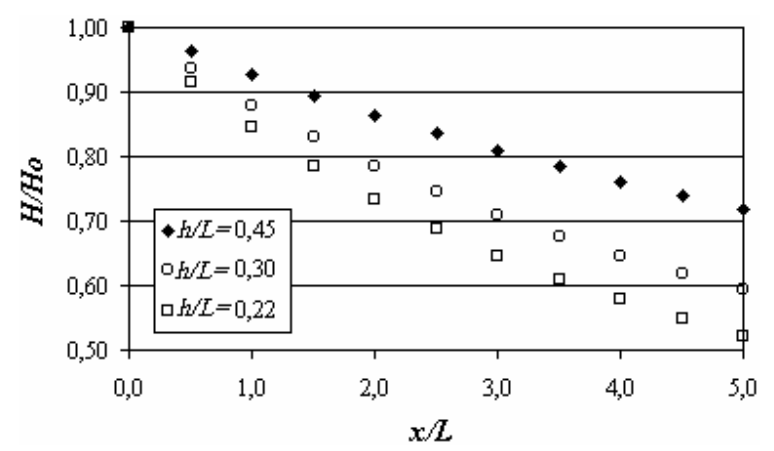

Figura 13 - Decaimento da altura da onda propagando-se através de um campo de hastes flexíveis com espaçamento $E_{\mathrm{v}}=0,025 \mathrm{~m}$.

\section{CONGLUSÕES}

Apresentou-se um modelo de amortecimento de ondas de gravidade propagando-se através de um conjunto de elementos artificiais flexíveis. O fenômeno foi dividido em dois processos principais. Ini-cialmente, a resistência individual de uma haste foi caracterizada através de um modelo mecânico. Uma vez estimada a contribuição de um elemento, avaliou-se o amortecimento gerado pelo conjunto, o que permitiu a quantificação de uma resistência adicional resultante do processo de interação entre hastes.

Dentro das simplificações consideradas, o modelo mecânico proposto mostrou-se satisfatório na representação da resposta da haste às forças impostas pelo escoamento. Uma vez calibrado, pôde-se gerar séries temporais de força, posição e velocidade ao longo da haste, o que caracteriza uma ferramenta útil para estudos físicos e biológicos. Acredita-se que a inclusão dos termos de inércia na equação do cálculo das forças hidrodinâmicas pode corrigir as defasagens temporais registradas em algumas das simulações. A investigação dos efeitos decorrentes da inclusão destes termos, juntamente com o aprimoramento da metodologia para obtenção dos coeficientes de calibração, são pontos a serem explorados em trabalhos futuros.

Para as características de onda e de haste analisadas, a resistência total do campo mostrou-se em média quatro vezes superior à soma das resistências individuais de cada haste. É possível que esta amplificação se deva à grande flexibilidade dos elementos estudados, pois fenômenos adicionais, como o entrelaçamento das hastes e o contato direto, não são considerados no modelo mecânico. Contudo, a quantificação deste fenômeno só pôde ser realizada graças à analise compartimentada do amortecimento, o que normalmente não é feito. Como a interação entre hastes pode ocorrer mesmo para elementos de maior rigidez (em conseqüência do desprendimento de vórtices, por exemplo) os resultados obtidos põem em dúvida a fundamentação teórica de grande parte dos modelos existentes, onde o fenômeno não é tratado explicitamente.

A expressão algébrica obtida para a curva de decaimento da altura da onda é semelhante à deduzida analiticamente para estruturas rígidas. $\mathrm{O}$ fator de amortecimento mostrou-se independente da altura da onda incidente, logo, mantidas as características físicas do campo, o decaimento pode ser expresso como uma função exclusiva da profundidade relativa. As expressões obtidas configuram uma ferramenta para dimensionamento das características físicas necessárias a um campo formado pelos elementos estudados, de modo que uma onda considerada nociva atinja padrões aceitáveis após percorrê-lo.

A metodologia e os resultados apresentados sugerem uma possibilidade para projeto de estrutura de amortecimento de ondas com base em um campo formado por espécies vegetais.

Uma vez caracterizada a resistência de um exemplar, uma primeira estimativa do potencial de amortecimento do campo poderia ser obtida pela expressão (2) considerando-se $\alpha=1$. Como os resultados indicam que em média este coeficiente tende a ser superior, pode-se dizer que o dimensionamento de um campo com o objetivo de atenuar ondas nocivas estaria sendo conservador, ou a favor da segurança. Estudos mais aprofundados poderiam ser conduzidos com o intuito de medir precisamente o amortecimento em um campo desta espécie, o que indicaria o potencial de amplificação decorrente da interação entre indivíduos.

\section{AGRADECIMENTOS}

Este trabalho foi desenvolvido com recursos do Projeto CAPES/PROCAD 0144/01-0 - "Amigos de Boussinesq - Rede Cooperativa de Pesquisa sobre Hidrodinâmica Costeira de Águas Rasas”, envolvendo a COPPE/UFRJ, a FEIS/UNESP e o I- 
$\mathrm{PH} /$ UFRGS. Os autores agradecem especialmente ao INPH - Instituto de Pesquisas Hidroviárias, onde os ensaios experimentais foram realizados, e a Moacyr de Paula, do NCE/UFRJ, pelo desenvolvimento do software de processamento das imagens.

\section{REFERÊNCIAS}

BREBBIA, C.A., WALKER, S., 1979, Dynamic analysis of offshore structures. 1 ed. London, NewnesButterworths.

CHAKRABARTI, S. K., 1987, Hydrodynamics of offshore structures. 1 ed. New York, Springer-Verlag.

COTTER, D.C., CHAKRABARTI, S.K., 1984, "Wave force tests on vertical and inclined cylinders". Journal of Waterways, Port., Coastal and Ocean Engineering, v. 110, n. 1, pp. 1-14.

DALRYMPLE, R. A., KIRBY, J. T., HWANG, P.A., 1984, "Wave refraction due to areas of energy dissipation". Journal of Waterways, Port., Coastal and Ocean Engineering, v. 110, n. 1, pp. 67-79.

DEAN, R. G., 1968, Relative validities of water wave theories. In: Technical Progress Report No. 16, University of Florida, Gainesville.

KOBAYASHI, N., RAICHLEN, A. W., ASANO, T., 1993, "Wave attenuation by vegetation". Journal of Waterways, Port, Coastal and Ocean Eng. Div., v.119, n.1, pp. 30-48.

LIMA, S.F., 2005. Amortecimento de ondas gravitacionais por campos de vegetação simulados por elementos de grande flexibilidade. Tese de Doutorado. Programa de Engenharia Oceânica, COPPE/UFRJ.

MENDEZ, F. J., LOSADA, I. J., 2004, "An empirical model to estimate the propagation of random breaking and nonbreaking waves over vegetation fields". Coastal Engineering, v. 51, pp. 103-118.

SARPKAYA, T., 1984. Discussion and closure on "Quasi-2-D forces on a vertical cylinder in waves" por Stansby, P.K., Bullock, G.N., Short, I., Journal of Waterways, Port, Coastal and Ocean Eng. Div., v.110, n.1, pp. 120-123.

STANSBY, P.K., BULLOCK, G.N., SHORT, I., 1983. "Quasi-2$\mathrm{D}$ forces on a vertical cylinder in waves". Journal of Waterways, Port., Coastal and Ocean Engineering, v. 109, n. 1, pp. 128-132.

VASCO, J.R.G., 2005, Modelo conceitual de dissipação da energia da onda que se propaga por fundos vegetados. Dissertação de Mestrado. Programa de PósGraduação em Engenharia Civil, Faculdade de Engenharia de llha Solteira, UNESP. 93p.

WIEGEL, R.L., 1964, Oceanographical Engineering. PrenticeHall.
ZDRAVICOVICH, M.M., 1984. Discussion and Closure on "Wave force coefficients on rough vertical cylinders", por S.K Chakrabarti, Journal of Waterways, Port, Coastal and Ocean Eng. Div., v.110, n.1, pp. 100104.

\section{Decay of Gravitational Waves by Fields of Flexible Vegetation. Part II - Experiments and Modelling}

\begin{abstract}
Gravity wave damping is treated by an innovative approach, which avoids the simplifying assumptions adopted by other models in the literature, which are not applicable to highly flexible plants. In order to verify the model, tests were conducted on a wave flume, using flexible, buoyant, $6 \mathrm{~mm}$ nylon ropes, fixed at a metallic grid on the bottom. A video technique for measuring displacement of the ropes was especially developed for calibrating the model. Tests were conducted for different wave conditions and spacing between ropes. By use of dimensional analysis, the experimental results provided an expression which relates the mean force on the stem to dimensionless parameters of wave and stem characteristics. This expression was inserted on the time averaged, depth integrated horizontal momentum equation, where an additional term for the reacting force of the stem was introduced. As a result, the wave decay along the field could be determined, and it became evident the importance of stem interaction on total wave height damping.

Key-words: gravitational waves; height damping.
\end{abstract}

